\title{
Article \\ Dark matter - Are they insulators to electromagnetic radiation?
}

\author{
Sayan Bag ${ }^{1}$ and Arijit Bag ${ }^{2, *(D)}$ \\ 1 Department of Physics, Sahid Matangini Hazra Govt. College for Women, West Bengal, India ; e-mail: \\ sayanbag08@gmail.com \\ 2 Department of Applied Science, Maulana Abul Kalam Azad University of Technology West Bengal, India; \\ e-mail: arijit.bag@makautwb.ac.in, bagarijit@gmail..com \\ * Correspondence: e-mail: arijit.bag@makautwb.ac.in, bagarijit@gmail..com; Tel.: +91-9735219451
}

Citation: Bag, S.; Bag, A. Dark matter - Are they insulators to electromagnetic radiation?. Preprints 2022, 1, 0. https://doi.org/

Publisher's Note: MDPI stays neutral with regard to jurisdictional claims in published maps and institutional affiliations.

Copyright: (C) 2022 by the authors Licensee MDPI, Basel, Switzerland. This article is an open access article distributed under the terms and conditions of the Creative Commons Attribution (CC BY) license (https:// creativecommons.org/licenses/by/ $4.0 /)$.

\begin{abstract}
The detection of Dark Matter is the greatest outstanding problem in modern cosmology. Several attempts have been taken for this without any remarkable success. To find out a suitable way of detection we need to understand its nature comprehensively. In the present article, a hypothesis is described considering Dark Matter as a normal matter. Its peculiar behavior is explained considering its existence in BEC state in the coolest part of the universe that makes it an electromagnetic insulator. Depending upon this hypothesis an experimental verification method is proposed.
\end{abstract}

Keywords: Dark matter; Bose-Einstein Condensation; Electromagnetic insulator

\section{Introduction}

Detection of Dark Matter [1,2] is one of the greatest outstanding problems of presentday fundamental physics. This is because the nature of the so-called Dark Matter is still unknown to the world of particle physics and cosmoparticle physics. Importantly, understanding the nature of Dark Matter and detection of its existence is the prime concern of cosmologists to justify Standard Models (SM) of our universe.

According to the SM, ordinary matter contributes less than $20 \%$ of the total matter of the universe [3]. The remaining part of the budget matter is unknown to us and considered as the Dark Matter. In the standard model of cosmology, the matter is considered as an entity that follows the relation of its energy density with its length scale as $\rho \propto a^{-3}$, where $a$ is the scale factor. For radiation, energy density is inversely proportional to the fourth power of the scale factor $\left(\rho \propto a^{-4}\right)$. In contrary to this, energy density remains constant during the change of the scale factor for any cosmological constant [3]. In SM, space is considered as cosmological constant. But, till now, such relation is not proved for Dark Matter. All possibilities are still open for it. It may be the same kind of entity as any kind of entity among these three or it may be a new kind of entity that follows a different relation. The answer to this question will help a lot to understand Dark Matter. Thus, we may verify the effect of Dark Matter on the cosmological model taking fictitious relationships of energy density and scale factor for it. These fictitious relationships of energy density and scale factor must include three above-mentioned relationships also.

In different ways, researchers are trying to overcome this problem. One group of people, disbelievers of Dark Matter, are trying to restructure the cosmological models by modifying the theory of gravity such that there would be no need for it to describe our universe comprehensively. But, day by day, new experimental results are obtained which are strongly corroborating the existence of Dark Matter. Thus, other groups of researchers are trying to elaborate the nature of Dark Matter by postulating new elementary particles like axions [4]. A successful $\Lambda C D M$ model requires the existence of Dark Matter [5]. Without its presence, the structure formation of the early Universe would not be possible according to the Big Bang model. The structure would not be reproducible also [6].

Lord Kelvin [7] is the first man who estimated the existence of dark bodies when he tried to explain the observed results of velocity dispersion of the stars that are orbiting around the center of the Milky Way galaxy with the estimated results from the theory. Later, in 1906, French mathematician Henri Poincaré used the term Dark Matter to discuss Kelvin's work, though there was a suspicion of a similar kind of entity long back in 1922 
by Dutch astronomer Jacobus Kapteyn [8]. This idea was nurtured by several groups of astronomers $[9,10]$ with the advancement of astronomy and cosmology research and inventions of high-resolution measurement techniques. The observation of gravitational lensing of background objects by galaxy clusters [11] gave a strong boost in favor of the existence of Dark Matter.

Dark matter researchers are tried to set up new directors to capture it. Different methodologies are followed. But all of these attempts have come out unsuccessful. This is because of the lack of sufficient knowledge about the nature of Dark Matter. It is obvious that without detecting a thing its properties could not be characterized properly. And, at the same time, it is also true that the scarcity of appropriate characterization of an entity designing its detector is like finding the needle in Khan's pile. Thus, the Dark Matter problem is like the chicken egg problem so far.

The only feature of Dark Matter is known to us is that it has high gravitational force but it is non-luminescence, i.e. it does not interact with electromagnetic radiation. Depending upon this little knowledge about Dark Matter, we may proceed in two different ways. We can formulate different mathematical models of universe postulating a few properties of Dark Matter and then by comparing theoretical results with experimental results we can judge whether our postulates about Dark Matter are true or not. This way of finding is like laying fruit by throwing stones. There is full of uncertainty with a chance of accidental success. On another path, we may postulate possibilities of properties of Dark Matter and find a suitable way to test it depending upon its postulated nature. In this method, we can at least eliminate a few possibilities or find out a few properties. Thus, this approach may be fruitful. The present way of detection of Dark Matter considering it as axion [4] falls in this category. The present research work is designed following this philosophy. In this investigation, Dark Matter is considered as a normal matter with some special features which are responsible for its abnormal gesture.

\section{Postulates}

Among a lot of possibilities about the nature of Dark Matter, it is very easy to consider it as similar to normal matter because the properties of normal matter are quite well known. If we could explain the reason behind its abnormal phenomenon as we have experienced for Dark Matter, our problem will be solved. This approach is not examined so far. Normal matter shows gravity. Thus, if we could explain its darkness, i.e. non-interaction with electromagnetic radiation, our job will be done.

To understand the non-interaction of Dark Matter - radiation interaction, we have to analyze how matter - radiation interactions take place. When radiation interacts with matter, three different incidents may occur - absorption of radiation, the reflection of radiation and emission of radiation. Reflection and emission make the object directly visible. The absorption of radiation makes the object hot and is followed by thermal emission. Thus, it is possible to detect the object by a thermal detector. To remain undetectable under radiation, these three processes should be shut down by the object.

Let us find how these three processes could be shut down by Dark Matter. If it absorbs radiation completely, there would not be any reflection, i.e., the object would behave like a perfect black body. But, a perfectly black body is a very good emitter. If we consider that emission is not possible, then heating will be there. Hence, our present job is to find out an appropriate way to stop emissions as well as heating.

In the case of normal matter, emission takes place due to the deexcitation of excited electrons. Thus, to be an emission-less object, possibilities of any kind of excitation should be ceased. May it be true for Dark Matter? Yes, It may be. How? It is known that the so-called Dark Matter only exists in the coolest part of the universe where the temperature is very close to $0 \mathrm{~K}$. Thus, there is a high possibility of Bose-Einstein condensate of these particles. The BEC of a huge amount of particles will lead to a state where there would be an infinite energy gap between the ground state and the excited state. Not only that at this state as all particles behave like a single particle its rest mass will be infinite and hence 
change of its vibrational state by finite radiation energy would not be possible. Thus, there would not be any heating of it. This condition may make Dark Matter a non-luminescence or non-interacting particle.

\section{Discussions}

Our postulates satisfy that a normal matter may behave like Dark Matter if an infinite number of such particles undergoes Bose-Einstein condensation. This is possible at a very low-temperature region of the universe. Thus, we can say that the Dark Matter is an insulator to electromagnetic radiation. Due to the presence of BEC, the mass density of Dark Matter will be high enough to exhibit a good gravitational attraction force.

If we consider Dark matter as described above, then how could we detect them? In reply to this question, it could be prescribed that if we disintegrate them by any means of way, we would be able to detect them. If we measure the total observable mass before and after the explosion of a black hole near any Dark Matter dominated region of the universe, a change of observable mass will indicate the correctness of this postulate.

\section{Conclusions}

In the present article Dark Matter is treated as a normal matter which exists in BEC state in the universe. Due to the condensation of an infinite number of particles, its effective rest mass becomes infinite which makes it an electromagnetic insulator. Considering this phenomenon of Dark Matter, a detection method is prescribed also. Future experimental verification may reveal the correctness of this hypothesis.

Author Contributions: Both the authors discussed to make this hypothesis.

Funding: No funding is obtained for this work.

Institutional Review Board Statement: Not required.

Informed Consent Statement: Not required.

Data Availability Statement: There is no hidden data.

Conflicts of Interest: It is Declared that therer is no conflicts of interest.

\section{Abbreviations}

The following abbreviations are used in this manuscript:

SM Standard Model

\section{References}

1. Arkani-Hamed, N., et al. A theory of dark matter. Physical Review D 2009,79.1, 15014.

2. Smith, P. F., and Lwin J. D.. Dark matter detection. Physics Reports 1990, 187.5, 203-280.

3. Pietro D. G. The Xenon Road to Direct Detection of Dark Matter at LNGS: The XENON Project. Universe 2021, 7.8, 313.

4. Backes, K. M., et al. A quantum enhanced search for dark matter axions. Nature 2021, 590.7845, $238-242$.

5. Frieman, J.; Turner, M.; Huterer, D. Dark Energy and the Accelerating Universe. Ann. Rev. Astron. Astrophys. $2008,46,385-432$.

6. Kolb, E.W.; Turner, M.S. The Early Universe; CRC Press: Boca Raton, FL, USA, 1990; Volume 69

7. Kelvin, L. (1904). Baltimore Lectures on Molecular Dynamics and the Wave Theory of Light. London, England.

8. Kapteyn, J. C. First attempt at a theory of the arrangement and motion of the sidereal system Astrophysical Journal 1922, 55, 302-327.

9. Oort, J.H. The force exerted by the stellar system in the direction perpendicular to the galactic plane and some related problems Bulletin of the Astronomical Institutes of the Netherlands 1932, 6, 249-287.

10. Zwicky, F. Die Rotverschiebung von extragalaktischen Nebeln Helvetica Physica Acta 1933, 6, 110-127.

11. Matthias B. Gravitational lensing Classical and Quantum Gravity 2010, 27.23, 233001. 